\title{
Efecto de tres fungicidas químicos en el control del Exserohilum spp. del maíz Chuska INIA 617
}

\section{Effect of three chemical fungicides in the control of Exserohilum spp. in corn Chuska INIA 617}

\author{
Patricia Delgado Campos ${ }^{1}$, Malluri Goñas Goñas ${ }^{1}$, Marielita Arce Inga ${ }^{1}$, Santos Triunfo Leiva Espinoza ${ }^{1}$ y Manuel Oliva ${ }^{1 *}$
}

\section{RESUMEN}

El objetivo del presente trabajo fue determinar el efecto de tres fungicidas químicos y tres dosis de aplicación para el control del "tizón foliar", Exserohilum spp., en el cultivo de maíz variedad INIA 617 Chuska, en el distrito de Levanto, Chachapoyas, Amazonas. Se empleó un diseño experimental en bloque completo al azar (DBCA) con tres repeticiones, donde se plantearon diez tratamientos, con un testigo sin ninguna aplicación. Los fungicidas y dosis fueron recomendados por los fabricantes: Pyraclostrobin + Epoxiconazole, con dosis 1=0,75 1/ha, dosis 2=1,125 1/ha, dosis 3=0,375 1/ha; Azosistrobim, con dosis 1=0,2 kg/ha, dosis 2=0,3 kg/ha, dosis 3=0,375 1/ha; y Sulfato de cobre + cal agrícola, con dosis $1=1 \mathrm{~kg} / 1001$ de agua, dosis $2=1,5 \mathrm{~kg} / 1001 \mathrm{de}$ agua, dosis $3=0,5 \mathrm{~kg} / 1001 \mathrm{lde}$ agua. El caldo bordelés se aplicó de manera preventiva 60 días después de la siembra. Los resultados se procesaron utilizando el software "R"; mediante un ANOVA y posteriormente una prueba Tukey, se encontró que el tratamiento T1 (testigo) fue el más afectado por la enfermedad, y el T7 como el menos atacado. Finalmente se demostró que el ingrediente activo Azoxistrobim, en sus tres dosis de aplicación, contribuye a disminuir y controlar el tizón foliar en maíz.

Palabras claves: Exserohilun sp, Pyraclostrobin, Epoxiconazole, Azosistrobim, sulfato de cobre, Amazonas.

\begin{abstract}
The objective of the present work was to determine the effect of three chemical fungicides and three application rates for the control of "leaf blight", Exserohilum spp., In the cultivation of variety INIA 617 Chuska maize in the district of Levanto, Chachapoyas, Amazonas . A randomized complete block experimental design (DBCA) was used with three replicates, where ten treatments were proposed, with one control without any application. Fungicides and doses were recommended by the manufacturers: Pyraclostrobin + Epoxiconazole, with doses $1=0.751 /$ ha, dose $2=1,125$ $1 /$ ha, dose $3=0,3751 /$ ha; Azosistrobim, with dose $1=0.2 \mathrm{~kg} /$ ha, dose $2=0,3 \mathrm{~kg} /$ ha, dose $3=0,3751 /$ ha; And copper sulfate + agricultural lime, with doses $1=1 \mathrm{~kg} / 1001$ water, dose $2=1.5 \mathrm{~kg} / 1001$ water, dose $3=0.5 \mathrm{~kg} / 1001$ water. The Bordeaux broth was applied preventively 60 days after sowing. The results were processed using software "R"; Through a ANOVA and later a Tukey test, T1 treatment (control) was found to be the most affected by the disease, and T7 as the least attacked. Finally, it was shown that the active ingredient Azoxystrobin, in its three application rates, contributes to the reduction and control of leaf blight in maize.
\end{abstract}

Keywords: Exserohilun sp, Pyraclostrobin, Epoxiconazole, Azosistrobim, copper sulphate, Amazon. 


\section{INTRODUCCIÓN}

El maíz (Zea mays L.) se cultiva en casi todo el mundo y ocupa el tercer lugar detrás del arroz y el trigo en cuanto a producción mundial total (FAS-USDA, 2005). Una de las principales razones de su alta producción es que es altamente demandado como alimento de diversos animales. En el Perú es frecuente la alimentación del ganado vacuno utilizando productos balanceados y forraje verde que proviene de la alfalfa y de variedades de maíz que en su mayoría son genéticamente degeneradas (INIA, 2013), con baja productividad, que contribuyen a elevar los costos y no permite que los ganaderos sean competitivos, afectando el consumo promedio de carne y leche (George et al., 2004).

El desarrollo de cultivares de maíz amarillo duro con aptitud forrajera en el Perú se encuentra en una etapa primaria. Sin embargo, en Estados Unidos, Argentina, Brasil y Uruguay, el forraje de maíz es el principal insumo para la producción de ganado (Moraes et al., 2014). El uso de forraje permite bajar los costos de producción de las proteínas y vitaminas de origen animal que constituyen la carne y la leche (Wilkinson, 2011). El consumo per cápita de carne en el Perú es de 10 kilos/persona/año, en comparación con Argentina y Brasil, que es de 40 y 60 kilos/año, respectivamente (INIA, 2013).

Las especies vegetales de interés forrajero se encuentran comprendidas en la familia de las gramíneas (maíz, avena, cebada, centeno, sorgo, mijo, etc.) y de las leguminosas (alfalfa, trébol, guisantes). Además, se incluyen algunas especies de raíces como las que pertenecen a las familias Quenopodiáceas, Crucíferas y Umbelíferas (CIMMYT, 2011)

La variedad de maíz forrajero INIA 617 Chuska es una variedad sintética de maíz forrajero conformada por nueve líneas con alto nivel de endogamia, que fueron recombinadas entre ellas. Es el resultado de los trabajos de investigación desarrollados por los investigadores y técnicos del Programa Nacional de Innovación Agraria en maíz de las Estaciones Experimentales Agrarias Vista Florida (Chiclayo), y El Porvenir (Tara- poto) (INIA, 2013).

El tizón foliar (Exserohilum turcicum), según condiciones climáticas, se presenta con niveles moderados a altos de severidad en maíces de siembra tardía y de segunda en el norte de la provincia de Buenos Aires. Su incremento entre otros factores estaría asociado a la continuidad y aumento de la superficie bajo siembra directa, a cambios y ampliación de la fecha de siembra (Couretot et al., 2012), a lotes con riego por aspersión (Formento, 2010), y también a frecuentes e intensas precipitaciones durante los meses estivales (Formento, 2010; Díaz, 2011).

En base a esta problemática existente en algunas áreas del Departamento Amazonas, se decidió evaluar el efecto de tres fungicidas químicos en el control del Exserohilum spp. "tizón foliar" del maíz INIA 617 Chuska en Quipachacha, distrito de Levanto (provincia de Chachapoyas).

\section{MATERIALY MÉTODOS}

\section{Área de estudio}

La presente investigación se desarrolló en el distrito de Levanto (provincia de Chachapoyas), a una altitud de 2400 m.s.n.m. aproximadamente

\section{Diseño de contrastación de la hipótesis}

Se trabajó con un Diseño en Bloque Completamente al Azar(DBCA).

\section{Población y muestra}

La población utilizada se compuso de plantas de maíz variedad INIA 617 Chuska, cultivadas bajo las condiciones de la estación experimental de Quipachacha (distrito de Levanto).

Se evaluaron tres plantas de maíz por unidad experimental tomadas de los dos surcos centrales para evitar un efecto de borde.

Métodos, técnicas e instrumentos de recolección de datos

Para las actividades de preparación de la parcela experimental, se realizó el reconocimiento del terreno, la limpieza de malezas, el arado e incorporación de los desechos vegetales. Posteriormente se dejó 25 días sin trabajar para la descomposición de los desechos. 
Finalmente, se pasó la rastra para el desmenuzado del suelo, delimitación y trazado del terreno de acuerdo al diseño.

\section{Tamaño del área experimental}

El área total del terreno que se usó fue de $602 \mathrm{~m}^{2}$; se consideraron tres bloques de experimentación, los cuales estuvieron conformados por 10 parcelas demostrativas con una distancia de separación entre bloques y parcelas de 1,0 m. De esta manera, cada parcela o unidad experimental tuvo un área de $18 \mathrm{~m}^{2}$ (6 x $3 \mathrm{~m})$.

En conclusión, cada unidad experimental estuvo conformada por una parcela de seis surcos, donde se eva- luaron tres plantas de acuerdo a la muestra calculada.

\section{Análisis de suelo}

Para realizar el análisis del suelo se tomaron las muestras a una profundidad de $30 \mathrm{~cm} 30$ días antes de la siembra.

\section{Fungicidas químicos}

El desarrollo de la investigación se fundamentó en la aplicación de tres fungicidas químicos, con tres dosis de aplicación, basándose en el laboratorio de cada fungicida. La dosis 1 fue según la recomendación del laboratorio; la dosis 2 fue sumando el 50\% de la recomendación; y la dosis 3 restando el 50\% de la recomendación del laboratorio (Tabla 1).

Tabla 1. Dosis de fungicidas químicos

\begin{tabular}{cccc}
\hline Dosis / Fungicida & Azoxistrobin & $\begin{array}{c}\text { Pyraclostrobin } \\
\text { Epoxiconazole }\end{array}$ & $\begin{array}{c}\text { Sulfato de cobre }+ \\
\text { cal agrícola }\end{array}$ \\
\hline Laboratorio (dosis 1) & $0,2 \mathrm{~kg} / \mathrm{ha}$ & $0,75 \mathrm{~L} / \mathrm{ha}$ & $1 \mathrm{~kg} / 100 \mathrm{~L}$ de agua \\
+ el 50\% (dosis 2) & $0,3 \mathrm{~kg} / \mathrm{ha}$ & $1,125 \mathrm{~L} / \mathrm{ha}$ & $1,5 \mathrm{~kg} / 100 \mathrm{~L}$ de agua \\
- el 50\%(dosis 3) & $0,1 \mathrm{~kg} / \mathrm{ha}$ & $0,375 \mathrm{~L} / \mathrm{ha}$ & $0,5 \mathrm{~kg} / 100 \mathrm{~L}$ de agua \\
\hline
\end{tabular}

Frecuencia de aplicación de los fungicidas.

\section{Pyraclostrobin + Epoxiconazole}

La frecuencia de aplicación para el presente fungicida fue tres veces durante toda la ejecución del ensayo, la primera aplicación se realizó a los 60 días después de la siembra, luego por las frecuentes lluvias la enfermedad persistía por lo que se procedió a realizar la segunda aplicación a los 80 días, posteriormente se realizó la última aplicación a los 90 días después de la siembra.

\section{Azoxistrobin}

La frecuencia de aplicación para Azoxistrobin fue de tres veces durante toda la ejecución del ensayo, la primera aplicación se realizó a los 60 días después de la siembra, luego por las frecuentes lluvias la enfermedad persistía por lo que se procedió a realizar la segunda aplicación a los 80 días, posteriormente se realizó la última aplicación a los 90 días después de la siembra.

\section{Sulfato de Cobre + Cal agrícola "caldo bórdeles"}

El caldo bórdales se aplicó como producto de preventivo, la primera aplicación se realizó a los 45 días después de la siembra, la frecuencia de aplicaciones se hizo semanalmente. El número de aplicaciones durante el desarrollo del cultivo fueron de 14 semanas.

\section{Tratamientos}

Para el desarrollo de la presente investigación se utilizaron tres fungicidas químicos, con tres dosis de aplicación. Estos tratamientos se describen en la Tabla 2

Tabla 2. Descripción de los tratamientos en estudio

\begin{tabular}{|c|c|}
\hline Tratamiento & Descripción \\
\hline $\mathrm{T} 1$ & Testigo \\
\hline $\mathrm{T} 2$ & $\begin{array}{c}\text { Pyraclostrobin }+ \\
\text { Epoxiconazole, } 0,75 \mathrm{~L} / \mathrm{ha}\end{array}$ \\
\hline $\mathrm{T} 3$ & $\begin{array}{c}\text { Pyraclostrobin }+ \\
\text { Epoxiconazole, } 1,125 \mathrm{~L} / \mathrm{ha}\end{array}$ \\
\hline $\mathrm{T} 4$ & $\begin{array}{c}\text { Pyraclostrobin }+ \\
\text { Epoxiconazole, } 0,375 \mathrm{~L} / \mathrm{ha}\end{array}$ \\
\hline T5 & Azoxistrobin $0,2 \mathrm{~kg} / \mathrm{ha}$ \\
\hline T6 & Azoxistrobin $0,3 \mathrm{~kg} / \mathrm{ha}$ \\
\hline $\mathrm{T} 7$ & Azoxistrobin $0,1 \mathrm{~kg} / \mathrm{ha}$ \\
\hline $\mathrm{T} 8$ & $\begin{array}{c}\text { Caldo bordelés } 1 \mathrm{~kg} / 100 \mathrm{~L} \mathrm{de} \\
\text { agua }\end{array}$ \\
\hline T9 & $\begin{array}{c}\text { Caldo bordelés } 1,5 \mathrm{~kg} / 100 \mathrm{~L} \\
\text { de agua }\end{array}$ \\
\hline $\mathrm{T} 10$ & $\begin{array}{c}\text { Caldo bordelés } 0,5 \mathrm{~kg} / 100 \mathrm{~L} \\
\text { de agua }\end{array}$ \\
\hline
\end{tabular}

\section{Evaluación}

De cada parcela o unidad experimental se evaluaron tres plantas. Estas evaluaciones fueron realizadas cada siete 
días, debido que esta enfermedad se propaga rápidamente.

Para evaluar el daño causado por Exserohilum sp., se registró como porcentaje de follaje infectado en plantas individuales, según la escala de Bleicher (1988). Cada planta evaluada fue considerada como un $100 \%$. La evaluación se inició 30 días después de la siembra; las aplicaciones de caldo bórdeles se realizaron de manera preventiva 45 días después de la siembra. Azoxistrobin y Pyraclostrobin junto con Epoxiconazole se aplicaron en presencia inmediata de la enfermedad tres veces en toda la etapa del cultivo.

\section{Cosecha}

La cosecha se realizó de forma manual, utilizando herramientas (machete y picadora), extrayendo todo el cuerpo, para posteriormente, picar toda la planta.

\section{RESULTADOS}

Nivel de incidencia de la enfermedad Exserohilum sp. “Tizón Foliar” en maíz INIA 617 Chuska.

Se observa que a los 124 días después de la siembra el tratamiento T7 (Azoxistrobin), con un 3\% de incidencia mostró el mejor resultado frente al tizón foliar, mientras que el tratamiento T1 (testigo) sin ninguna aplicación, fue el que mayor porcentaje de incidencia desarrolló, con un valor final de $25 \%$ (Tabla 3 ).

Tabla 3. Incidencia de Exserohilum sp "tizón foliar” en plantas de maíz

\begin{tabular}{ccccccccccc}
\hline Tratamiento / DDS & $\mathbf{6 1}$ & $\mathbf{6 8}$ & $\mathbf{7 5}$ & $\mathbf{8 2}$ & $\mathbf{8 9}$ & $\mathbf{9 6}$ & $\mathbf{1 0 3}$ & $\mathbf{1 1 0}$ & $\mathbf{1 1 7}$ & $\mathbf{1 2 4}$ \\
\hline T1 & 2 & 3 & 3 & 6 & 7 & 7 & 10 & 15 & 18 & 25 \\
T2 & 1 & 4 & 4 & 8 & 8 & 9 & 7 & 7 & 6 & 9 \\
T3 & 2 & 2 & 4 & 8 & 10 & 16 & 14 & 10 & 10 & 10 \\
T4 & 2 & 3 & 3 & 6 & 8 & 10 & 10 & 12 & 8 & 8 \\
T5 & 2 & 2 & 4 & 6 & 8 & 8 & 10 & 8 & 5 & 5 \\
T6 & 0 & 1 & 4 & 9 & 9 & 8 & 7 & 7 & 5 & 5 \\
T7 & 0 & 1 & 4 & 8 & 10 & 6 & 5 & 5 & 3 & 3 \\
T8 & 0 & 3 & 6 & 8 & 10 & 15 & 18 & 20 & 22 & 22 \\
T9 & 1 & 1 & 6 & 6 & 8 & 10 & 18 & 20 & 23 & 23 \\
T10 & 0 & 1 & 4 & 8 & 10 & 12 & 18 & 18 & 20 & 20 \\
\hline
\end{tabular}

DDS $=$ días después de la siembra

En el momento de la evaluación final, el tratamiento T7, T6 y T5 mostraron valores de 3, 5 y 5, respectivamente. Los tratamientos T2, T3, T4, T8, T9 у T10, alcanzaron valores de $6,10,8,22,23,20$, respectivamente, difiriendo estadísticamente del primero.

Tabla 4. Prueba de comparaciones múltiples para observar los niveles de incidencia del tizón foliar causado por Exserohilum sp. en plantas de maíz INIA 617 Chuska.

\begin{tabular}{ccccc}
\hline \multirow{2}{*}{ Tratamiento } & \multicolumn{2}{c}{ Evaluación Inicial (EV6) } & \multicolumn{2}{c}{ Evaluación Final (EV10) } \\
\cline { 2 - 5 } & Promedio & Grupo homogéneo & Promedio & Grupo homogéneo \\
\hline T1 & 7 & $\mathrm{AB}$ & 25 & $\mathrm{~A}$ \\
$\mathrm{~T} 2$ & 9 & $\mathrm{AB}$ & 9 & $\mathrm{AB}$ \\
$\mathrm{T} 3$ & 16 & $\mathrm{~A}$ & 10 & $\mathrm{AB}$ \\
$\mathrm{T} 4$ & 10 & $\mathrm{AB}$ & 8 & $\mathrm{AB}$ \\
T5 & 8 & $\mathrm{AB}$ & 5 & $\mathrm{C}$ \\
T6 & 8 & $\mathrm{AB}$ & 5 & $\mathrm{C}$ \\
T7 & 6 & $\mathrm{C}$ & 3 & $\mathrm{C}$ \\
T8 & 15 & $\mathrm{~A}$ & 22 & $\mathrm{~A}$ \\
T9 & 10 & $\mathrm{AB}$ & 23 & $\mathrm{~A}$ \\
T10 & 12 & $\mathrm{~A}$ & 20 & $\mathrm{~A}$ \\
\hline
\end{tabular}

En la tabla 4 se muestra que los tratamientos T8, T4 y T10, son aquellos que presentan mayor desarrollo de la enfermedad, mientras que el T7 mostró el menor desarrollo de la enfermedad después de la primera aplicación de fungicida. En la evaluación final se visualiza que existe diferencia significativa entre 
tratamientos, resultando de esta los tratamientos T7, T5, y T6, lo cual se traduce en que la aplicación del fungicida (Azoxistrobin) hizo efecto al reducir el \% de incidencia del tizón foliar en maíz.

\section{Área Bajo la Curva del Progreso de la Enfermedad (ABCPE) \%-días}

En la figura 1 se observa que en los tratamientos T7, T6 y T5 (Azoxistrobin), el tizón foliar alcanzó valores inferiores que en el resto de tratamientos $(>50)$. Por otro lado, el tratamiento T1, 101 días después de la siembra alcanzó el valor más alto (450) de área afectada por el tizón foliar, Finalmente, los tratamientos T8, T9 y T10, mostraron valores intermedios.

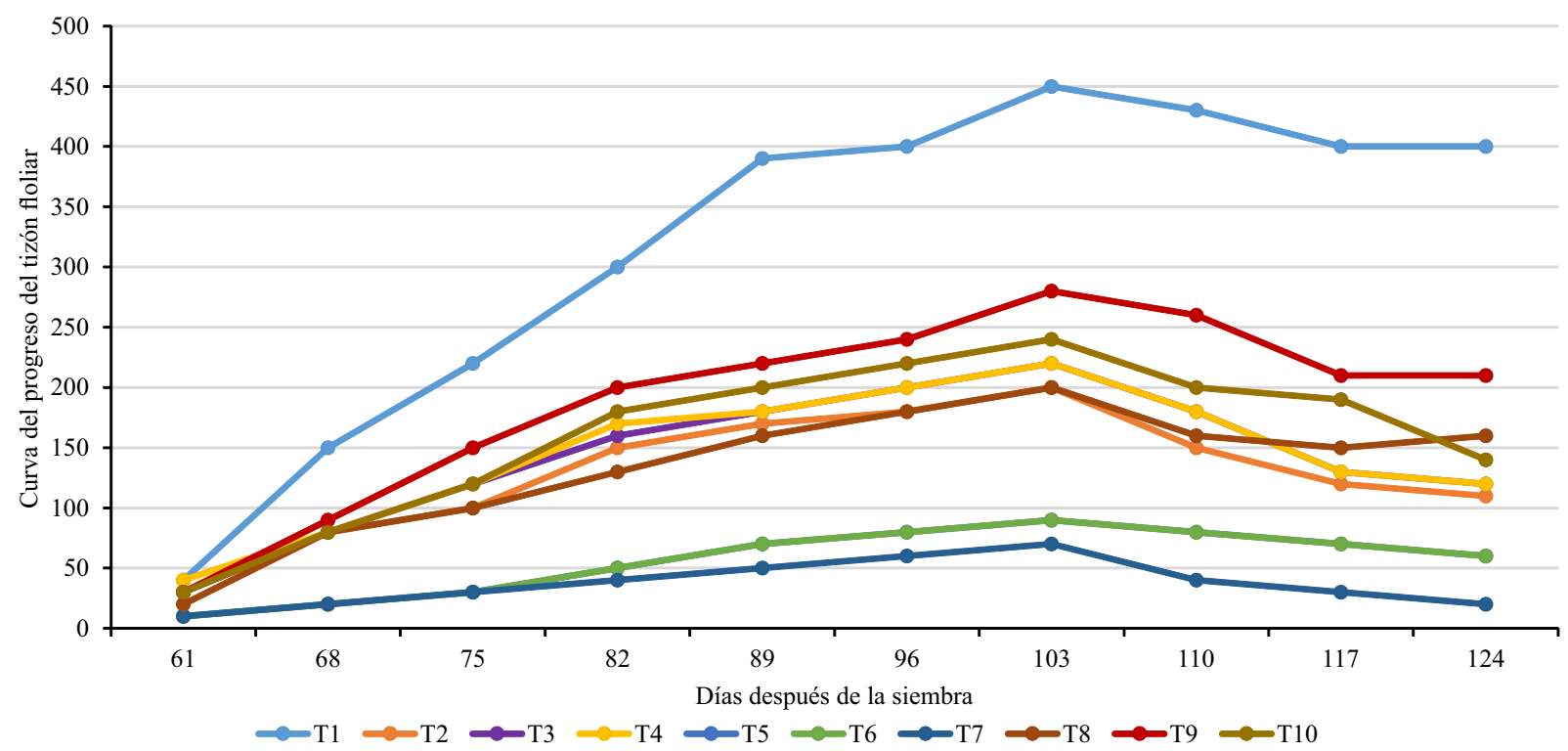

Figura 1. ABCPE por tratamientos en el ensayo realizado en Quipachacha.

En la tabla 5 se observan tres grupos bien definidos, donde el Tratamiento T1 (testigo), es el que presentó promedio de mayor incidencia, con un valor de 395,11 frente a la enfermedad. Por el contrario, los tratamientos T5 y T7, con aplicación de Azoxistrobin, son los que presentaron menor área afectada por la enfermedad, con 91,39 y 65,72 , respectivamente.

Tabla 5. Prueba de Comparación Múltiple de TUKEY al 0,05 para el promedio ABCPE del tizón foliar en plantas de maíz

\begin{tabular}{cc}
\hline Tratamiento & Promedio \\
\hline T1 & 395,11 \\
T2 & 118,61 \\
T3 & 124,06 \\
T4 & 122,50 \\
T5 & 91,39 \\
T6 & 94,11 \\
T7 & 65,72 \\
T8 & 161,00 \\
T9 & 190,56 \\
T10 & 193,61 \\
\hline
\end{tabular}

\section{Rendimiento de forraje verde (kg/ha)}

En la figura 2 se observa que el tratamiento T7, con
$85925 \mathrm{~kg} /$ ha alcanzó el mayor rendimiento de forraje verde por hectárea, sin embargo no difiere estadísticamente con el resto de tratamientos. En segundo lugar, la mejor ubicación en cuanto al rendimiento de forraje verde lo obtiene el tratamiento T4, con $80740 \mathrm{~kg} / \mathrm{ha}$, seguido de los tratamientos T5, T3, y T6. El tratamiento que alcanzó menor rendimiento fue el tratamiento $\mathrm{T} 10$ con $53333 \mathrm{~kg} / \mathrm{ha}$, seguido del tratamiento $\mathrm{T} 1$ (testigo) con $54074 \mathrm{~kg} / \mathrm{ha}$.

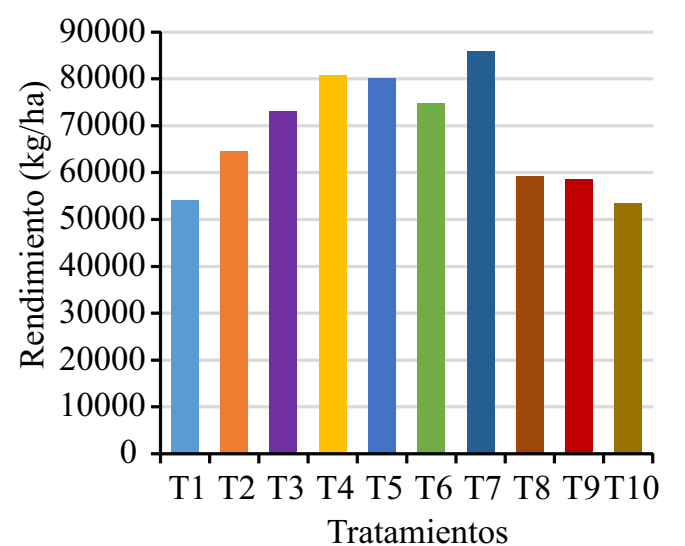

Figura 2. Rendimiento de forraje verde de maíz Chuska INIA 617. 


\section{Rendimiento de materia seca de maíz}

En la tabla 6 puede observar los valores que corresponden al porcentaje de materia seca respecto al peso de forraje verde (de 0,2 $\mathrm{kg}$ de forraje verde por tratamiento). Los resultados indican la ausencia de diferencias estadísitcas significativas entre los tratamientos establecidos, sin embargo, numéricamente se observa, que el tratamiento T7 alcanzó el mayor porcentaje de materia seca, con el 77\%, seguido del tratamiento T5, con el $75 \%$ de materia seca.

Tabla 6. Rendimiento de materia seca en \% en plantas de maíz, de acuerdo a los tratamientos establecidos en la investigación

\begin{tabular}{ccc}
\hline Tratamamiento & Promedio & M.S. (\%) \\
\hline T1 & 0.09 & $46 \%$ \\
T2 & 0.12 & $60 \%$ \\
T3 & 0.12 & $60 \%$ \\
T4 & 0.13 & $63 \%$ \\
T5 & 0.15 & $75 \%$ \\
T6 & 0.14 & $70 \%$ \\
T7 & 0.15 & $77 \%$ \\
T8 & 0.12 & $61 \%$ \\
T9 & 0.13 & $66 \%$ \\
T1 0 & 0.12 & $60 \%$ \\
\hline
\end{tabular}

\section{Rendimiento de materia seca (\%) de maíz INIA 617}

\section{Chuska}

En la figura 3 se observa que el tratamiento T7, con el $77 \%$, alcanzó el mayor rendimiento en materia seca de maíz INIA 617 Chuska. Por el contrario, el tratamiento $\mathrm{T} 1$ (testigo), alcanzó el $46 \%$ de rendimiento de materia seca, situándose en el otro extremo del porcentaje.

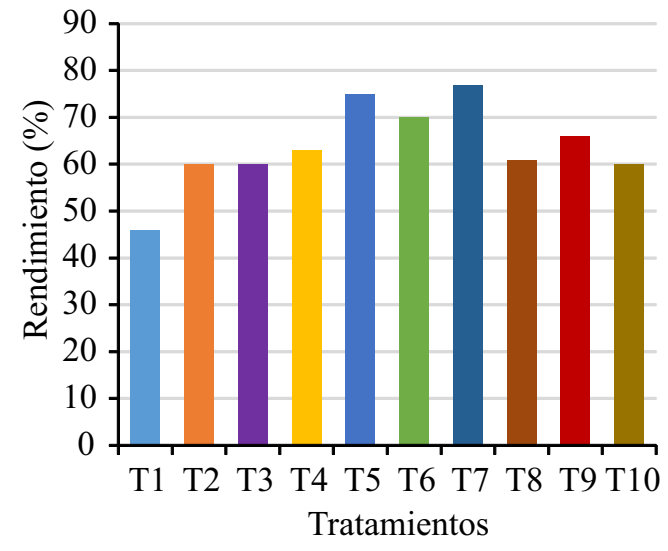

Figura 3. Rendimiento de materia seca.

\section{DISCUSIÓN}

Los altos niveles de incidencia encontrados en la presente investigación, se deben a que el fitopatógeno Exserohilun sp. causante de la enfermedad conocida como tizón foliar en plantas de maíz, se desarrolla en condiciones de temperaturas bajas, humedad alta y exceso de lluvias, generando así un microclima que favorece la diseminación del fitopatógeno (Araujo et al., 2001). En Quipachacha (Levanto), donde se ejecutó la investigación, se presentaron condiciones favorables para el desarrollo del hongo, por lo que los resultados muestran altos niveles de incidencia causados por el fitopatógeno. El resultado obtenido se corrobora con la investigación de Bert y Satorre (2014), quienes mencionan estas mismas condiciones climáticas en su investigación, con resultados similares.

El Área Bajo la Curva del Progreso de la Enfermedad (ABCPE), del tizón foliar (Exserohilum sp.) muestra sus valores más altos y se comportó acorde a las necesidades del fitopatógeno (Bertelsen et al., 2011). Se llegaron a obtener valores de 450 en la curva de área afectada, lo que indica un $90 \%$ de presencia de la enfermedad en la plantación de maíz. Lo que se corrobora con la investigación de Díaz (2011), quien menciona que el $\mathrm{ABCPE}$ puede llegar a obtener valores mayores de 400, valor que indica pérdidas por al menos un $90 \%$ de su producción.

Por otro lado y según el reporte de Cromey et al. (2014), en los últimos años se han informado efectos directos de fungicidas, especialmente del grupo de las estrobilurinas como Azoxistrobin y Pyraclostrobin, entre otros, con frecuencias de aplicación de tres veces, que contribuyeron a controlar las enfermedades del área foliar verde en gramíneas. Lo descrito anteriormente se asemeja a los resultados alcanzados, donde Azoxistrobin es la estrobilurina que mejor valor alcanzó dentro de la presente investigación, con tres veces durante todo el ciclo de vida del cultivo de maíz INIA 617 Chuska.

En el caso del caldo bórdeles, compuesto básicamente por cal agrícola y sulfato de cobre, no alcanzó a tener algún efecto represivo sobre el hongo Exserohilum sp. 
Al respecto, según el SENASA (2012), manifiesta que este producto es utilizado desde hace mucho tiempo, para curar enfermedades de las plantas, ya que actúa como un excelente fungicida, acaricida, y además actúa como fungicida preventivo en el caso de hongos, sobre todo del phylum Basidiomycota. Al respecto se muestra que el hongo Exserohilun sp en maíz, quien pertenece al phylum Ascomycota, no genera algún efecto significativo, por lo que no controla esta enfermedad del Tizón Foliar en maíz INIA 617 Chuska.

En la presente investigación también se encontró que el ingrediente activo Azoxistrobin reportó los mejores resultados en sus tres dosis de aplicación, lo encontrado es corroborado por Cabrera y Uchupailla (2011) quienes utilizaron 10 fungicidas con tres dosis de aplicación, basándose en recomendaciones del laboratorio, de tal forma que en condiciones de laboratorio obtuvieron que score $250 \mathrm{EC}$, fue uno de los tres productos químicos que obtuvo mejor resultado.

Los resultados encontrados podrían estar indicando que que el maíz INIA 617 Chuska, es un híbrido altamente susceptible a este hongo Exserohilum sp., y es que a mayor humedad y exceso de lluvias, el hongo se muestra más persistente, lo que puede llegar afectar toda la cosecha de maíz si no se toman medidas de prevención para que esta enfermedad pueda ser controlada (Seghers et al., 2004). Esto se corrobora en parte con los estudios de Fantin y Duarte (2012), donde muestran que en híbridos susceptibles, el control químico es un instrumento válido para el control del tizón foliar, de lo contrario este fitopatógeno puede llegar a matar todo el cultivo. Lo que podría indicar que sin la aplicación de algún producto químico, la producción no será adecuada (Dahmardeh et al., 2009).

Los resultados alcanzados en la presente investigación dan a mostrar además que el rendimiento en forraje del testigo $\mathrm{T} 1$ es de 54 t, lo que se traduce en una pérdida de forraje de $40 \mathrm{~kg} / \mathrm{ha}$, por lo que es necesario la aplicación de algún control químico. Al respecto, Couretot et al. (2012), determinaron el efecto de fungicidas foliares y momento de aplicación sobre la severidad de tizón foliar y enfermedades de raíz y tallo en maíz, y encontraron que la incidencia de tizón foliar afectó el rendimiento, con una pérdida de $40 \mathrm{~kg} / \mathrm{ha}$ por cada incremento de $1 \%$ de severidad. Aunque, también concluyeron que las mezclas de triazoles y estrobilurinas ensayadas fueron eficientes en el control de tizón foliar.

Finalmente, INIA (2013), menciona que el rendimiento potencial de forraje verde del maíz INIA 617 Chus$\mathrm{ka}$, es de $95 \mathrm{t} / \mathrm{ha}$, lo cual indica que en la presente investigación el Tratamiento T7 a base de Azoxistrobin, es el que mejor rendimiento reportó con un total de $86 \mathrm{t} /$ ha de forraje verde, los cuales se aproximan a los resultados obtenidos dentro del ensayo. Estos resultados similares a los teóricamente originales, sin presencia de enfermedad, son los adecuados a través del uso de agroquímicos (Bradley y Ames, 2010), quedando claro que su uso disminuye la incidencia y la severidad de este fitopatógeno (Flaherty y Dunkle, 2005).

\section{CONCLUSIONES}

El mejor efecto de control químico de Exserohilum sp., agente causal del tizón foliar, fue alcanzado por el fungicida a base de Azoxistrobin.

Para el Área Bajo la Curva del Progreso de la Enfermedad (ABCPE), el tratamiento más afectado fue el T1, con mayor área con presencia de enfermedad, seguido del T9, T2, T10, T3, T8 y T4. Por el contrario, los tratamientos T7, T5 y T6 reportaron menor área afectada por la presencia de Exserohilum sp.

El tratamiento T7, a base de Azoxistrobin, con una dosis de $0,1 \mathrm{~kg} / \mathrm{ha}$, es el que mejor resultado obtuvo, concluyendo que el ingrediente activo Azoxistrobin es una estrobilurina que en sus tres dosis de aplicación, controla el tizón foliar en maíz INIA 617 Chuska. Para el rendimiento de forraje verde, los mejores resultados se dieron en los tratamientos T7, T5 y T6, mientras que el más bajo se dio en el T1 (testigo absoluto). Lo que indica que los niveles de tizón foliar del maíz afectaron los rendimientos del follaje. 


\section{REFERENCIAS BIBLIOGRÁFICAS}

Araujo, W. L., W. Maccheroni, C.I. Aguilar-Vildoso, P. A. V. Barroso, H. O. Saridakis, y J. L. Azevedo. 2001. "Variability and interactions between endophytic bacteria and fungi isolated from leaf tissues of citrus rootstocks". Can.J. Microbiol.47:229-236.

Bert, F. E., y E. Satorre. 2014. Fecha de siembra y el cultivo de maíz temprano y tardio. Producción de maíz. Rosario (Argentina): AAPRESID.

Bertelsen J. R., E. de Neergaard E., y V. SmedegaardPetersen. 2011. "Fungicidal effects of azoxystrobin and epoxiconazole on phyllosphere fungi, senescence and yield of winter". Plant Pathology 50: 190-205.

Bleicher, J. 1998. Niveis de resistencia a Helminthosporium turcicum Pass. em tres ciclos de selecao em milho pipoca (Zea mays L.). Tesis de Doctorado. Universidade de São Paulo. Sao Paulo (Brasil).

Bradley, C. A., y K. A. Ames. 2010. "Effect of foliar fungicides on corn with simulated hail damage". Plant Disease 94: 83-86.

Cabrera, A. G., y J. V. Uchupailla. 2011. Evaluación de 10 fungicidas en el control de Botrytis cinerea Pers. en el cultivo de fresa (Fragaria virginiana Var. Diamante) a nivel de laboratorio. Tesis de Grado. Universidad de Cuenca. Cuenca (Ecuador).

CIMMYT. 2011. Enfermedades del maíz: una guía para su identificación en el campo. México D.F. (México): CIMMYT. Recuperado de: http://repository.cimmyt.org/xmlui/bitstream /handle/10883/812/94349.pdf

Couretot L., L. Parisi G. Ferraris, y G. Magnone. 2012. "Efecto de fungicidas foliares y momento de aplicación sobre la intensidad de tizón foliar y enfermedades de raíz y tallo". En Actas XIV Jornadas Fitosanitarias. Buenos Aires (Argentina)

Cromey M.G., R.C. Butler M.A. Mace, y A.L.J. Cole.
2014. "Effects of the fungicides azoxystrobin and tebuconazole on Didymella exitialis, leaf senescence and grain yield in wheat". Crop Protection 23: 1019-1030.

Dahmardeh, M., A. Ghanbari, B. Syasar, y M. Ramroudi. 2009. "Effect of intercropping maize (Zea mays L.) with cow pea (Vigna unguiculata L.) on green forage yield and quality evaluation". Asian journal of plant sciences 8: 235-241.

Díaz, C. 2011. Principales enfermedades del maíz en Tucumán y su manejo. Tucumán (Argentina): A A P R E S I D. R e c u p e r a d o d e : http://www.maizar.org.ar/documentos/sinto matologia\%20y\%20manejo\%20de\%20enfer medades\%20del\%20maiz\%20en\%20region $\% 20$ noa.pdf

Fantin G. M., y A.P. Duarte. 2012. Manejo de doencas na cultura milho safrinha. Sao Paul (Brasil): Instituto Agronomico Camoinas.

FAS-USDA. 2005. Crop Production Tables: World grains by commodity. Washington DC (EEUU): World Agricultural Production.

Flaherty, J. E., y L. D. Dunkle. 2005. "Identification and expression analysis of regulatory genes induced during conidiation in Exserohilum turcicum”. Fungal Genetics and Biology 42: $471-481$.

Formento, A.N. 2010. Enfermedades foliares reemergentes del cultivo de maíz: royas (Puccinia sorghi y Puccinia polysora), tizón foliar (Exserohilum turcicum) y mancha ocular (Kabatiella zeae). Buenos Aires (Argentina): INTA.

George, C., W. P. Ridley, J. C. Obert, M. A. Nemeth, M. L. Breeze, y J. D. Astwood. 2004. "Composition of grain and forage from corn rootworm-protected corn event MON 863 is equivalent to that of conventional corn (Zea mays L.)". J. Agric. Food Chem. 52: 41494158.

INIA (Instituto Nacional de Innovación Agraria). 
2013. Ficha técnica de maíz forrajero INIA

617 Chusca. Chiclayo (Perú): INIA.

Recuperado de: http://www.inia.gob.pe/prodservicios/publicaciones/publicacion/tripticos /item/205-09-2010-maiz-forrajero-inia-617chuska

Moraes, A., P. C. de Faccio Carvalho, I. Anghinoni, S. B. C. Lustosa, S. E. V. Andrade, y T. R. Kunrath. 2014. "Integrated crop-livestock systems in the Brazilian subtropics". European Journal of Agronomy 57: 4-9.

Seghers, D., L. Wittebolle, E. M. Top, W. Verstraete, y S. D. Siciliano. 2004. "Impact of agricultural practices on the Zea mays L. endophytic community". Applied and Environmental Microbiology 70: 1475-1482.

SENASA (Servicio Nacional de Salud Animal). 2012. Manejo integrado de la roya amarilla del cafeto. San Martín (Perú): SENASA. R e c u p e r a d o d e : http://www.oroverde.com.pe/pdf/manual_ro ya.pdf

Wilkinson, J. M. 2011. "Re-defining efficiency of feed use by livestock". Animal 5: 1014-1022. 